\title{
Fixpunktthermometer für kleine Rohrquerschnitte
}

\author{
Augustin, Silke ${ }^{1}$; Fröhlich, Thomas ${ }^{1}$; Marin, Sebastian ${ }^{1}$; Breitkreutz, Paul ${ }^{1}$; Lehmann, Harald ${ }^{2}$ \\ 1 TU IImenau, Fakultät für Maschinenbau, Institut für Prozessmess- und Sensortechnik, 98693 \\ Ilmenau; Tel.-Nr. +49 3677/69 1487, E-Mail-Adresse: silke.augustin@tu-ilmenau.de \\ 2 electrotherm Gesellschaft für Sensorik und thermische Messtechnik mbH, Gewerbepark 6, 98716 \\ Geraberg; Tel.-Nr. +49 3677/7956 17, E-Mail-Adresse: h.lehmann@electrotherm.de
}

\section{Zusammenfassung}

Am Institut für Prozessmess- und Sensortechnik der TU IImenau wurden in Kooperation mit der Firma electrotherm Geraberg GmbH seit mehreren Jahren selbstkalibrierende Thermometer für den Einsatz in konventionellen Kraftwerken entwickelt und erfolgreich erprobt. Einige Sensoren haben dabei mehr als 1000 Kalibrierzyklen durchlaufen, ohne dass signifikante Veränderungen der Fixpunkt-Temperaturen nachweisbar sind. Rekalibrierungen ausgesuchter Sensoren im Kalibrierlabor nach vierjähriger Einsatzzeit zeigten, dass der tatsächliche Wert der FixpunktTemperatur nur um max. 0,15 K gegenüber dem Neuzustand verändert war. Die Zuverlässigkeit und Präzision der Messmethode wurde für den Langzeiteinsatz nachgewiesen.

Heißdampf-Rohrleitungen in den Kraftwerksanlagen haben zumeist Nenndurchmesser von 200$600 \mathrm{~mm}$. Bei dezentraler Energieerzeugung sind zumeist kleinere Rohrquerschnitte $(40 \ldots 100 \mathrm{~mm})$ für den jeweiligen Wärmeträgertransport üblich (solarthermische Anlagen, Blockheizkraftwerke, ...). Aber auch hier sind höchstmögliche Prozesstemperaturen wünschenswert, um entsprechende Wirkungsgrade zu erzielen. Will man in diesen Rohrleitungen Fixpunkt-Thermometer (mit ihren Miniaturfixpunkttiegeln von wenigstens $25 \mathrm{~mm}$ Länge) zum Einsatz bringen, besteht das Dilemma entweder darin, inakzeptabel große Strömungsverluste oder andernfalls bei zu geringer Einbautiefe eine Verfälschung der Thermoelement-Temperatur durch Umgebungstemperatureinflüsse zu erzeugen. Das hier vorliegende Anwendungsbeispiel bezieht sich auf den Einsatz eines selbstkalibrierenden Thermoelements in einem Wärmeträgerkreislauf im Temperaturbereich mit einer Maximaltemperatur von $400{ }^{\circ} \mathrm{C}$ und einer Strömungsgeschwindigkeit von $0,2 \ldots 1,8 \mathrm{~m} / \mathrm{s}$.

Im vorliegenden Beitrag wird aufgezeigt, wie mit Hilfe numerischer Berechnungen und experimenteller Untersuchungen die Miniatur-Fixpunkt-Thermometer für diese Einsatzfälle optimiert werden.

\section{Einleitung}

Am Institut für Prozessmess- und Sensortechnik der TU Ilmenau wurden in Kooperation mit der Firma electrotherm Geraberg $\mathrm{GmbH}$ selbstkalibrierende Thermoelemente (SKTE) u.a. für den Einsatz in Kraftwerken entwickelt [1, 2]. Die mit einem geeigneten Fixpunktmaterial gefüllte Keramikzelle wird von einem Heizelement umschlossen, mit dem die Innentemperatur der Zelle $\vartheta_{U}(t)$ über die Temperatur des Messmediums angehoben werden kann (Abbildung 1). 

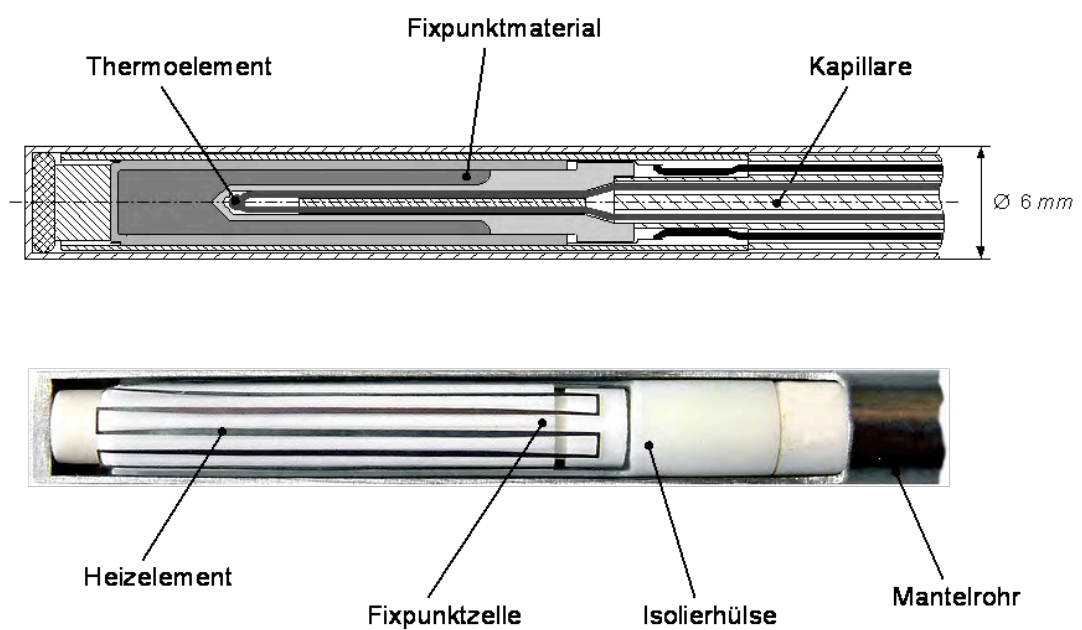

Abbildung 1: Aufbau eines SKTE für den industriellen Einsatz

Beim Erreichen der Schmelztemperatur $\vartheta_{L P}$ des Fixpunktmaterials wird aufgrund der thermischen Effekte der Phasenumwandlung durch das Thermoelement ein gegenüber der

Umgebungstemperatur der Zelle $\vartheta_{U}(t)$ zeitlich verzögerter Temperaturverlauf $\vartheta_{T E}(t)$ registriert (siehe Abbildung 2). Aus dem Verlauf von $\vartheta_{T E}(t)$ bzw. $U\left[\vartheta_{T E}(t)\right]$ wird über spezielle Auswerteverfahren ein Kalibrierwert $U\left(\vartheta_{L P}\right)$ gewonnen, anhand dessen das Thermoelement und die angeschlossene Messkette unter Betriebsbedingungen kalibriert/nachjustiert werden können [3].

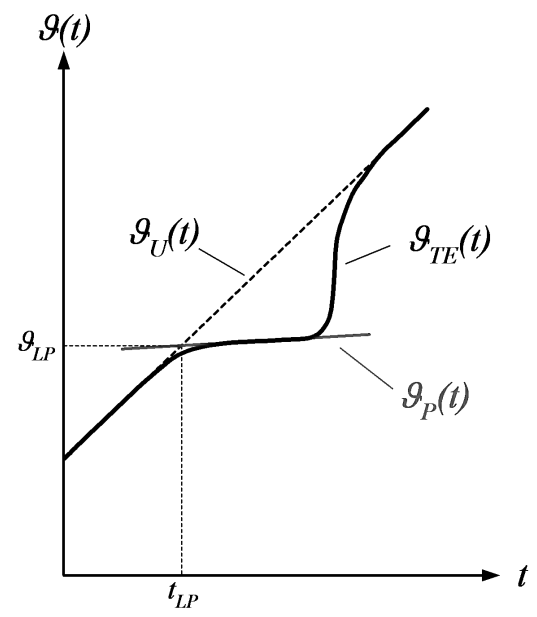

Abbildung 2: Zeitlicher Temperaturverlauf in der miniaturisierten Fixpunktzelle

Seit 2004 sind Fixpunkt-Thermoelemente kommerziell verfügbar, die speziell für den Kraftwerkseinsatz konzipiert wurden [4]. Während der Heißdampf $\left(535-545^{\circ} \mathrm{C}\right)$ konventionell mit Thermoelementen gemessen wird und dabei eine Messunsicherheit von bis $\mathrm{zu}+/-5 \mathrm{~K}$ besteht, gelingt mit der Fixpunkt-Methode eine Reduzierung auf $+/-1 \mathrm{~K}$ (Abbildung 3). Damit darf die Prozesstemperatur um $4 \mathrm{~K}$ näher an die Auslegungstemperaturgrenze der Anlage gefahren werden, was mit einem höheren Wirkungsgrad und einem geringeren $\mathrm{CO}_{2}$-Ausstoß je erzeugter Kilowattstunde verbunden ist.

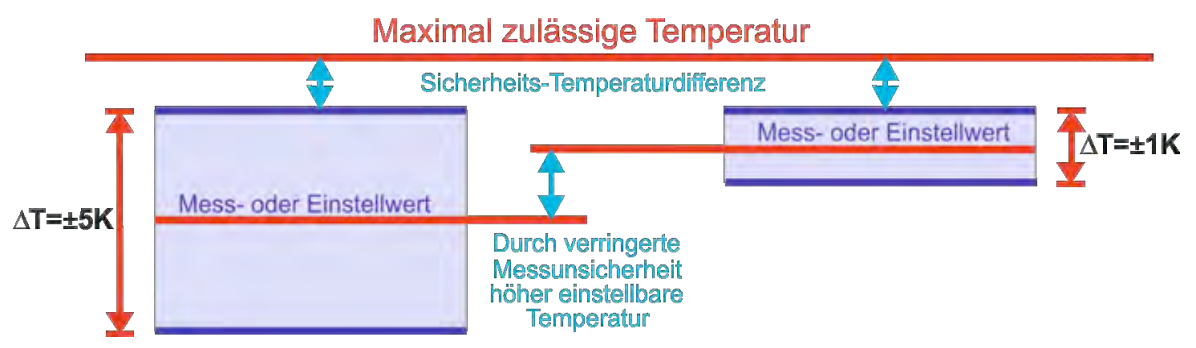

Abbildung 3: Erhöhung der maximalen Einsatztemperatur durch verringerte Messunsicherheit (in diesem Beispiel $4 \mathrm{~K}$ ) [10] 
In der folgenden Abbildung sind drei konventionelle Arbeitsthermoelemente zu sehen, die die Frischdampftemperatur im Kraftwerk messen. Durch das zusätzliche Fixpunkt-Thermoelement (2. Messstelle von rechts) wird deren Drift ständig überwacht und somit sichergestellt, dass die maximal zulässige Temperatur von hier $545^{\circ} \mathrm{C}$ sicher eingehalten wird [5].

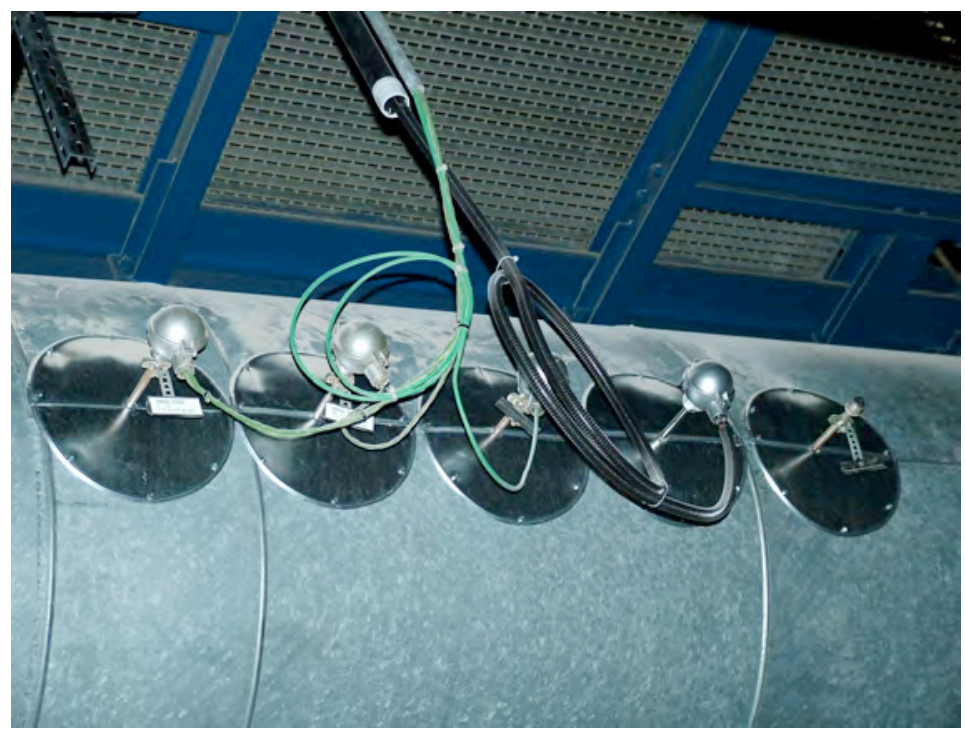

Abbildung 4: Typische Messstellen im Kraftwerkseinsatz

Solche Kraftwerksanwendungen laufen inzwischen seit mehreren Jahren bei Temperaturen von $535-545^{\circ} \mathrm{C}$. Einige Sensoren haben dabei mehr als 1000 Kalibrierzyklen durchlaufen, ohne dass signifikante Veränderungen der Fixpunkt-Temperaturen nachweisbar sind. Rekalibrierungen ausgesuchter Sensoren im Kalibrierlabor nach vierjähriger Einsatzzeit zeigten, dass der tatsächliche Wert der Fixpunkt-Temperatur nur um max. 0,15 K gegenüber dem Neuzustand verändert war. D.h., die Zuverlässigkeit und Präzision der Messmethode wurde für den Langzeiteinsatz nachgewiesen [5].

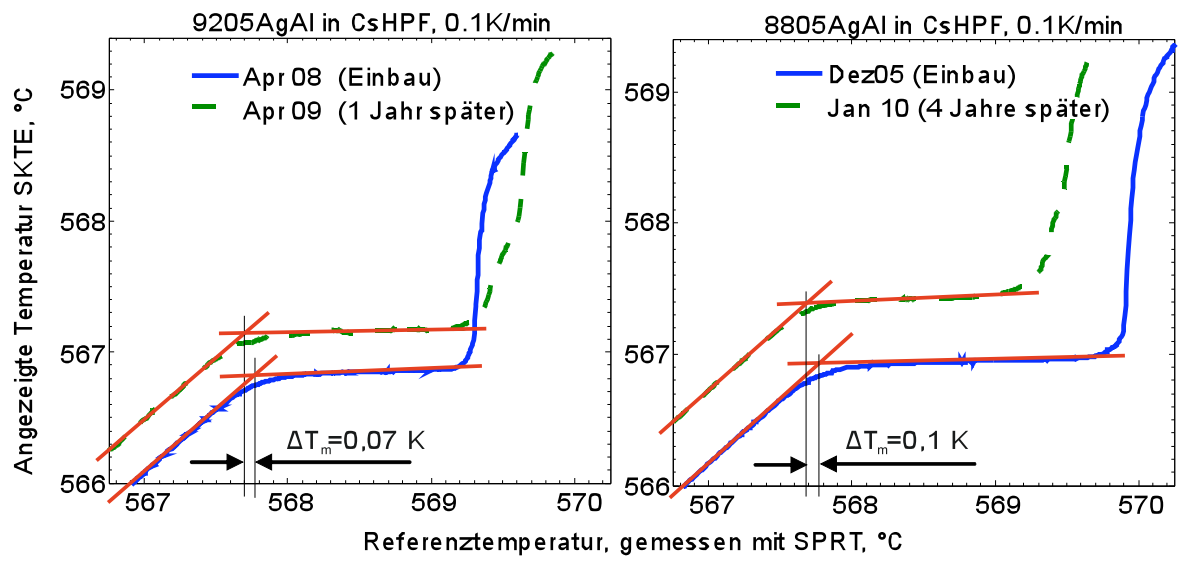

Abbildung 5: Schmelzplateaus zweier Fixpunktsensoren, die bei Kalibrierungen im Labor gegen ein

Präzisionswiderstandsthermometer (SPRT) in einem Cs-Wärmerohr-Ofen jeweils vor und nach Langzeiteinsatz im Kraftwerk registriert wurden. Obwohl die Höhe und Form der Graphen sich gegenüber dem jeweiligen Neuzustand verändern, sind die Abszissenwerte der Schnittpunkte hier max. um 0,1 K gewandert, d.h. der als Fixpunkt approximierte Schmelzplateau-Parameter ist stabil.

\section{Neue Anforderung: Kleine Rohrdurchmesser}

Heißdampf-Rohrleitungen in den Kraftwerksanlagen haben zumeist Nenndurchmesser von 200$600 \mathrm{~mm}$. Die ø 6mm-Messeinsätze der Fixpunktthermometer kommen in dort üblichen Schweißstutzen der Form 4 nach DIN EN 43772 zum Einsatz. Diese dickwandigen, konisch gestalteten Schutzrohre haben sich als Kompromiss von hoher mechanischer Festigkeit, 
kleinstmöglichem Strömungswiderstand und messtechnisch notwendiger Eintauchtiefe des Thermometers bewährt.

Bei dezentraler Energieerzeugung sind zumeist kleinere Rohrquerschnitte $(40 \ldots 100 \mathrm{~mm})$ für den jeweiligen Wärmeträgertransport üblich (solarthermische Anlagen, Blockheizkraftwerke, ...). Aber auch hier sind höchstmögliche Prozesstemperaturen wünschenswert, um entsprechende Wirkungsgrade zu erzielen. Also besteht auch bei diesen Anwendungsfällen die Zielstellung darin, den Messfehler soweit wie möglich zu minimieren, um so dicht wie möglich an die Auslegungstemperatur der jeweiligen Anlage heranzukommen.

Will man in diesen Rohrleitungen mit kleinem Querschnitt Fixpunkt-Thermometer (mit ihren Miniaturfixpunkttiegeln von wenigstens $25 \mathrm{~mm}$ Länge) zum Einsatz bringen, besteht das Dilemma entweder darin, inakzeptabel große Strömungsverluste oder andernfalls bei zu geringer Einbautiefe eine Verfälschung der Thermoelement-Temperatur durch Umgebungstemperatureinflüsse zu erzeugen. Wenn der statisch-thermische Messfehler Größenordnungen von 0,1 K und mehr annimmt, ist eine so genaue in-situ-(Fixpunkt-) Kalibrierung nicht mehr sinnvoll.

Deshalb wurde untersucht, welche konstruktiven Maßnahmen ergriffen werden könnten, um mit der vorhandenen Messeinsatz-Geometrie auch bei kleinen Rohrquerschnitten akzeptable Messergebnisse erzielen zu können. Der Lösungsansatz besteht in einem numerischen Modell auf Basis der Finiten Elemente Methode (FEM), das sich z.B. im Hinblick auf das verwendete Wärmetransport-Mittel (Heißdampf, Öl), Prozesstemperatur, Schutzrohrwerkstoff, Isoliermaterialien u.ä. leicht variieren lässt. Solch ein Modell kann immer dann zum Einsatz kommen, wenn z.B. Machbarkeitsstudien für zukünftige oder die Optimierung vorhandener Messstellen angefragt werden.

Das hier vorliegende Anwendungsbeispiel bezieht sich auf den Einsatz eines selbstkalibrierenden Thermoelements in einem Wärmeträgerkreislauf im Temperaturbereich mit einer Maximaltemperatur von $400{ }^{\circ} \mathrm{C}$ und einer Strömungsgeschwindigkeit von $0,2 \ldots 1,8 \mathrm{~m} / \mathrm{s}$. Anhand der kriteriellen Gleichungen in [9] wurde daraus ein Wärmeübergangskoeffizient $\alpha>2.000 \mathrm{~W} / \mathrm{m}^{2} \mathrm{~K}$ ermittelt. Als Fixpunktmaterial wurde Zink verwendet, dessen Schmelztemperatur $\vartheta=419,527^{\circ} \mathrm{C}$ beträgt. Bei der angenommen Mediumstemperatur von $400{ }^{\circ} \mathrm{C}$ muss der Phasenumwandlungsprozess durch Zuschalten der integrierten Heizung (siehe Abbildung 1) ausgelöst werden.

\section{Numerische Berechnungen}

Im Vorfeld des Einsatzes dieser Thermometer wurden numerische Berechnungen mit dem FEMProgrammsystem ANSYS Workbench durchgeführt, bei denen die Phasenumwandlung des Fixpunktmaterials berücksichtigt wurde. Der konstruktive Aufbau des Thermometers wurde für die Simulation im Rahmen des üblichen Pre-Processings für FEM-Berechnungen angepasst (Abbildung 6). So wurden kleinere Details wie z.B. Fasen entfernt und kleinere Bauteile, wie z.B. die TE-Drähte im Inneren zu einem Bauteil zusammengefasst [6]. Da von einem symmetrischen Aufbau der Rohrleitung und des Thermometers ausgegangen werden konnte, wurde das Modell im Halbschnitt erstellt (Einsparung von Rechenzeit und Speicherplatz). 


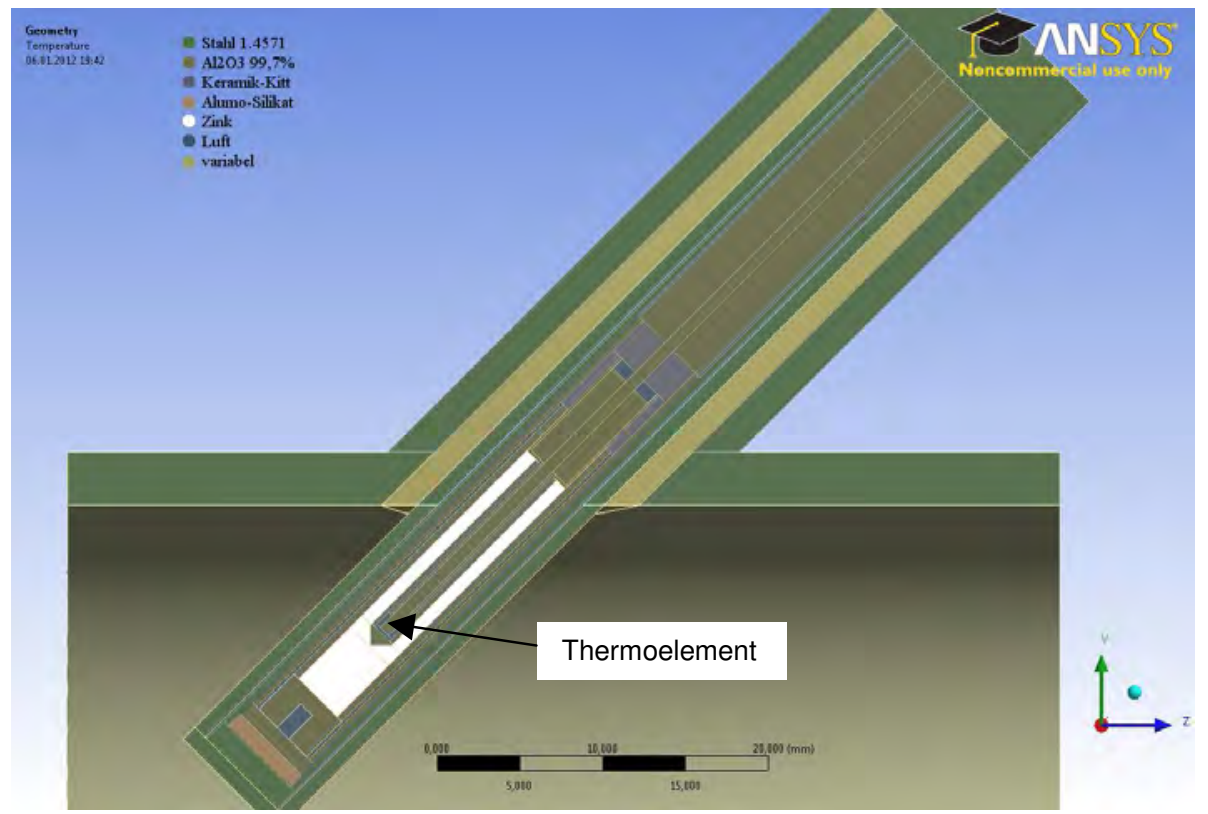

Abbildung 6: Konstruktiver Aufbau der Messstelle in kleinen Rohrleitungen

Das in Abbildung 6 als „variabel“ bezeichnete Bauteil wurde in den durchgeführten Berechnungen mit den Materialien Luft bzw. Stahl durchgeführt. Diese Variation war notwendig, um abschätzen zu können, welche Auswirkungen ein entsprechendes Spiel beim Einbau des Messeinsatzes in das fest eingebaute Schutzrohr der Rohrleitung haben würde.

Das folgende Bild zeigt die Vernetzung in Finite Elemente. Bei der gegebenen Problemstellung wurde eine transiente, itterative Rechnung mit Beschreibung der Phasenumwandlung durchgeführt.

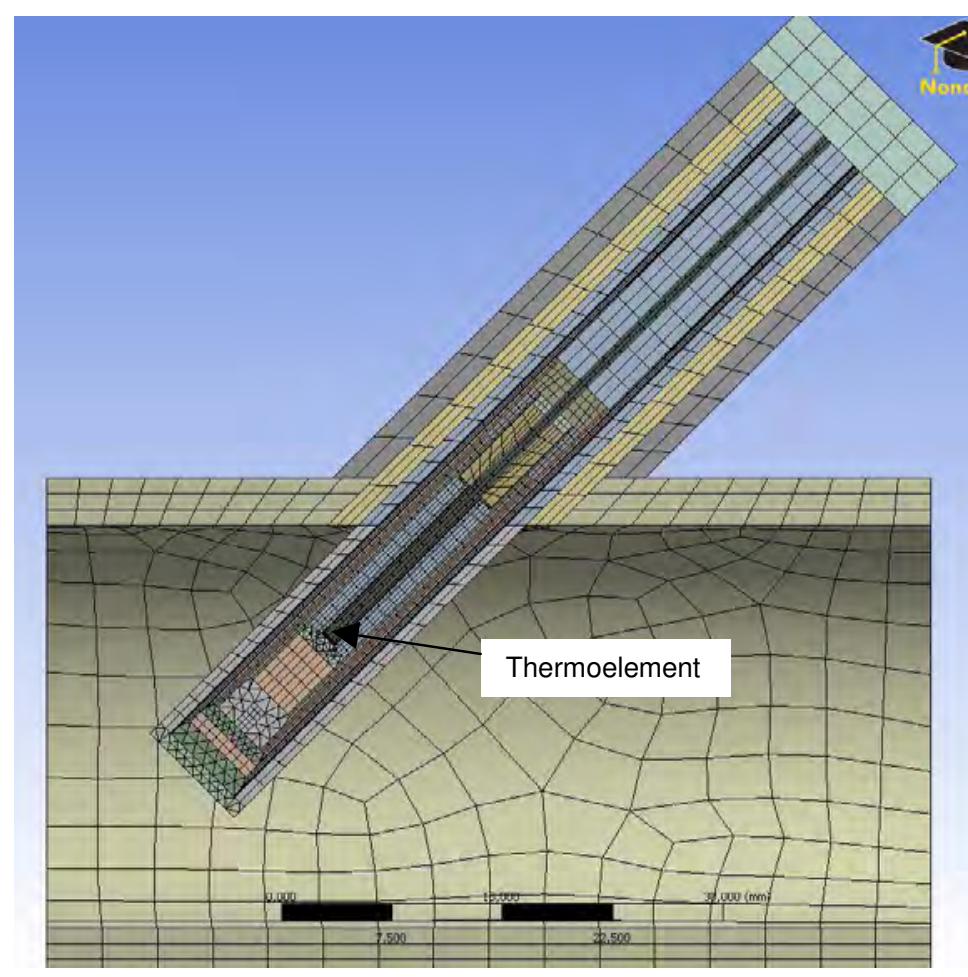

Abbildung 7: Finites Elemente Modell

Die Phasenumwandlung während der Schmelz- und Erstarrungsvorgänge kann durch eine sprunghafte Änderung der Enthalpie beschrieben werden. Die Enthalpie $H$ ist ein Maß für den 
Energiegehalt eines Systems und hängt von der inneren Energie $U$, dem Druck $p$ und dem Volumen $V$ ab [7]:

$$
H=U+p \cdot V
$$

Für das im vorliegenden Anwendungsfall verwendete Fixpunktmaterial Zink wurde die im folgenden Diagramm dargestellte Enthalpieänderung in die Berechnung eingefügt. In der vorliegenden Version des Programmsystems ANSYS Workbench kann die Eingabe der Enthalpie nicht direkt, sondern in Form eingefügter apdl-Kommandos erfolgen.

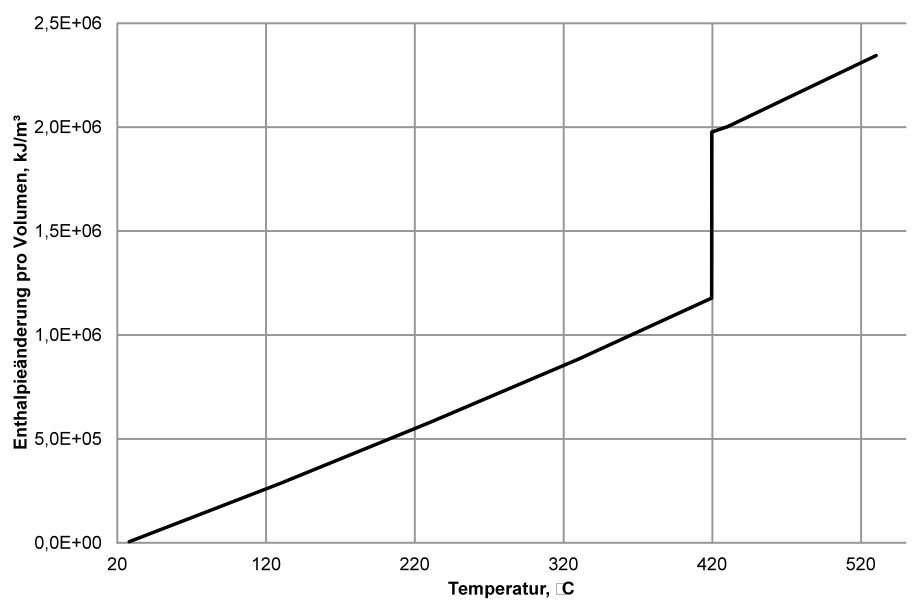

Abbildung 8: Enthalpieänderung für Fixpunktmaterial Zink

In den hier dargestellten FEM-Berechnungen wurde für die Innenwand der Rohrleitung sowie die Oberfläche des Thermometers im Einbaubereich ein Wärmeübergangskoeffizient $\alpha=2.000 \mathrm{~W} / \mathrm{m}^{2} \mathrm{~K}$ und eine Mediumstemperatur $\mathrm{T}_{\mathrm{M}}=400^{\circ} \mathrm{C}$ angenommen, für den Wärmeübergang an der Außenfläche der Rohrleitung und des Thermoemeters ein $\alpha=10 \mathrm{~W} / \mathrm{m}^{2} \mathrm{~K}$ und eine Umgebungstemperatur $\mathrm{T}_{U}=20^{\circ} \mathrm{C}$. Damit ist für die Kopplung zur Umgebung bezüglich der Auswirkungen auf den statisch-thermischen Messfehler der ungünstigste Fall angenommen worden, eventuell angebrachte Isolationsmaterialien verringern den Wärmetransport zur Umgebung und damit die Größe des statisch-thermischen Messfehlers.

Die numerische Berechnung erfolgte in zwei Schritten:

1. Berechnung des stationären Temperaturfeldes bei gegebenen Wärmeübergangsbedingungen (Ausgangstemperaturverteilung für Schritt 2)

2. Transiente Temperaturfeldberechnung (durch Zuschalten der Heizung $\rightarrow$ Auslösen der Phasenumwandlung).

Zunächst wurde eine stationäre Temperaturfeldberechnung mit den oben angegebenen Randbedingungen durchgeführt. Das Ergebnis dieser Berechnungen (Abbildung 9) bildete den Ausgangspunkt für den zweiten Berechnungsschritt, in dem durch ein Zuschalten der integrierten Heizung der Phasenumwandlungsprozess simuliert werden sollte.

Die Heizung wurde als auf die Oberfläche des Miniaturfixpunkttiegels (Abbildung 1) aufgebrachte Wärmestromdichte simuliert. Dabei wurde die Heizleistung rampenförmig auf eine Maximalgröße von $11 \mathrm{~W}$ mit einer Geschwindigkeit von ca. $1 \mathrm{~W} / \mathrm{min}$ erhöht. Dieses Heizregime wurde in den experimentellen Untersuchungen (siehe unten) als die günstigste Variante bestimmt, um durch langsames Erwärmen den Phasenumwandlungsprozess so zu gestalten, dass ein hinreichendes thermisches Gleichgewicht zwischen dem Phasenumwandlungsmaterial und dem Sensorelement gewährleistet ist. Um eine sichere Auswertung und Erkennung der Schmelztemperatur zu gewährleisten, sollten die gemessenen Phasenumwandlungsplateaus möglichst keine Neigung, keine Abweichung von der Waagerechten aufweisen und eine Mindestlänge nicht unterschreiten [7]. Bei den kleinen Miniatur-Fixpunktzellen können Umgebungseinflüsse und dadurch bedingte Wärmeleitungsvorgänge diese Plateauform und -dauer stark beeinflussen. Eine übliche Verfahrensweise ist es daher, Miniaturfixpunkt-Kalibrierungen unter quasi-stationären Bedingungen mit geringen Aufheizraten durchzuführen. 


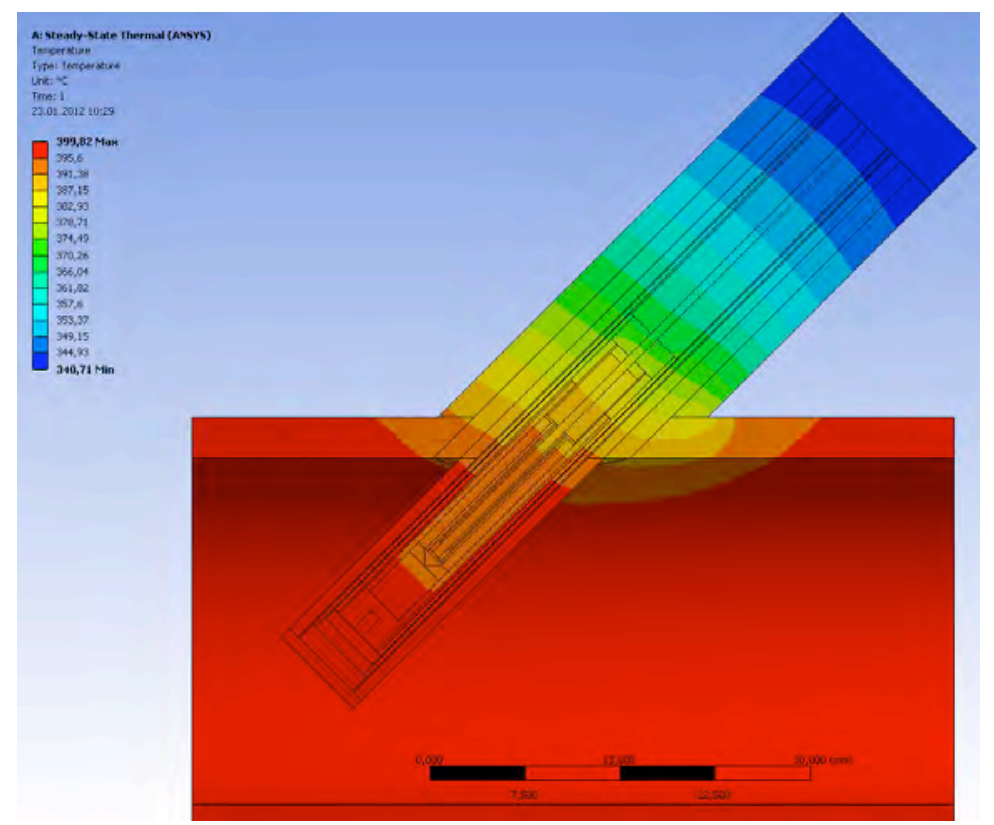

Abbildung 9: Temperaturverteilung als Ergebnis der FEM-Berechnung für den stationären Fall (variabler Teil Stahl)

Aus den stationären Berechnungen wurde der aufgetretene statisch-thermische Messfehler für die beiden Einbauvarianten mit Luft bzw. Stahl an der Spitze des Themoelements $\left(T_{S}\right)$ für $\mathrm{T}_{\mathrm{M}}=400{ }^{\circ} \mathrm{C}$ bestimmt.

$$
\Delta T=T_{S}-T_{M}
$$

Folgende Ergebnisse wurden für die beiden Möglichkeiten der variablen Materialbeschreibung im Einbaubereich in einer ersten Modellrechnung ermittelt:

$$
\begin{aligned}
& \Delta T_{\text {Luft }}=-3,6 \mathrm{~K} \\
& \Delta T_{\text {Stahl }}=-4,1 \mathrm{~K}
\end{aligned}
$$

Der größere Fehler bei dem angenommenen Fall eines bündigen Einbaus des Messeinsatzes in das Schutzrohr (variabler Teil mit Materialeigenschaft Edelstahl angenommen) ist erklärbar mit der damit verbundenen besseren Wärmeleitung zum Schutzrohr und damit zur Umgebung. Auch die Wärmetransportvorgänge innerhalb des Thermoemeters (entlang der Thermoelementdrähte, des Messeinsatzes und der verwendeten Keramiken) spielen eine große Rolle.

Insgesamt ist der berechnete statisch-thermische Messfehler zu groß, um die Temperatur in der Rohrleitung exakt bestimmen zu können. Wie oben erwähnt wird angestrebt, die Mediumstemperatur mit einer Genauigkeit von $0,1 \mathrm{~K}$ zu bestimmen. Man könnte natürlich den statisch-thermischen Messfehler durch einen in der Messwertverarbeitung impementierten Algorithmus korrigieren, wenn er einmal exakt für einen bestimmten Anwendungsfall sowohl numerisch als auch experimentell bestimmt werden konnte. Dann ist es aber wenig sinnvoll, den Aufwand des Einsatzes eines selbstkalibrierenden Thermometers zu betreiebn - die Gesamtunsicherheit der Messung ist viel zu groß. Aus diesem Grund muss die konstruktive bzw. materialtechnische Gestaltung der Thermometer auf jeden Fall durch künftige Berechnungen optimiert werden.

Auch im zweiten Berechnungsschritt wurde der Temperaturverlauf an der Spitze des Thermoelements ausgewertet, um den berechneten Verlauf der Temperatur während der Phasenumwandlung darzustellen. 


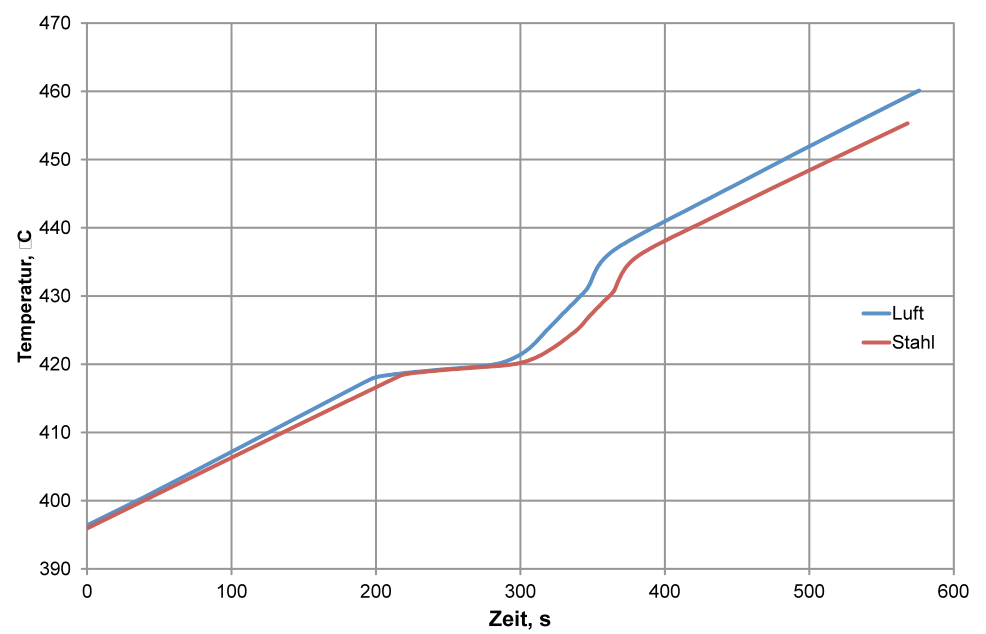

Abbildung 10: Temperaturverlauf an der Thermoelementspitze nach Zuschalten der Heizung

Erkennbar ist, dass der Temperaturanstieg bei einem direkten Kontakt zwischen dem Messeinsatz und dem Schutzrohr (Stahl) langsamer erfolgt und die Phasenumwandlung erst ca. 10 s später einsetzt. Erklärbar ist dieser Effekt wieder mit der stärkeren Wärmeableitung zum Schutzrohr und damit zur Umgebung.

Der Plateauverlauf selbst sieht in beiden Fällen ähnlich aus. Gegenüber dem in Abbildung 2 dargestellten theoretischen Verlauf sowie dem in Abbildung 5 dargestellten Verlauf einer typischen Messung im Kalibrierlabor ist im hier berechneten Fall kein gerader, scharfer Austritt aus dem Phasenplateau zu erkennen. Die Wärmeableitung innerhalb des Thermometers entlang der Thermoelementdrähte und der Keramikkapillare, die zur elektrischen Isolation der Thermoelementdrähte verwendet werden, beeinflusst den Plateauverlauf stark. Wie in [7] dargestellt, erfasst das Thermoelement einen Temperaturwert, der von sich in seinem Messort überlagernden Wärmeströmen bestimmt wird. Neben den Wärmeströmen zwischen Fixpunktmaterial und Thermoelement-Messort sind dies auch die Wärmeströme zwischen Messort, Messmedium und Umgebung. Schlußfolgernd daraus muss neben den oben aufgeführten Anforderungen an die Verringerung des statisch-thermischen Messfehlers die konstruktiven und materialtechnischen Optimierungen auch eine Verbesserung der Plateauform erreicht werden.

\section{Experimentelle Verifikation der Ergebnisse}

Die dargestellten numerischen Ergebnisse wurden für einen konkreten Anwendungsfall dargestellt. $\mathrm{Da}$ die Modellierung selbst, die Beschreibung der Randbedingungen, Kontaktflächen sowie der Materialdaten einer gewissen Unsicherheit unterliegen, wurde das numerische Modell durch Experimente verifiziert. Die Messungen konnten nicht an einem konkreten Einsatzfall (solarthermische Anlagen, Blockheizkraftwerke ...) durchgeführt werden. Deshalb wurden sie zunächst im Labor mit Hilfe eines Wasserkreislaufes den zu erwartenden Bedingungen angenähert (Abbildung 11).
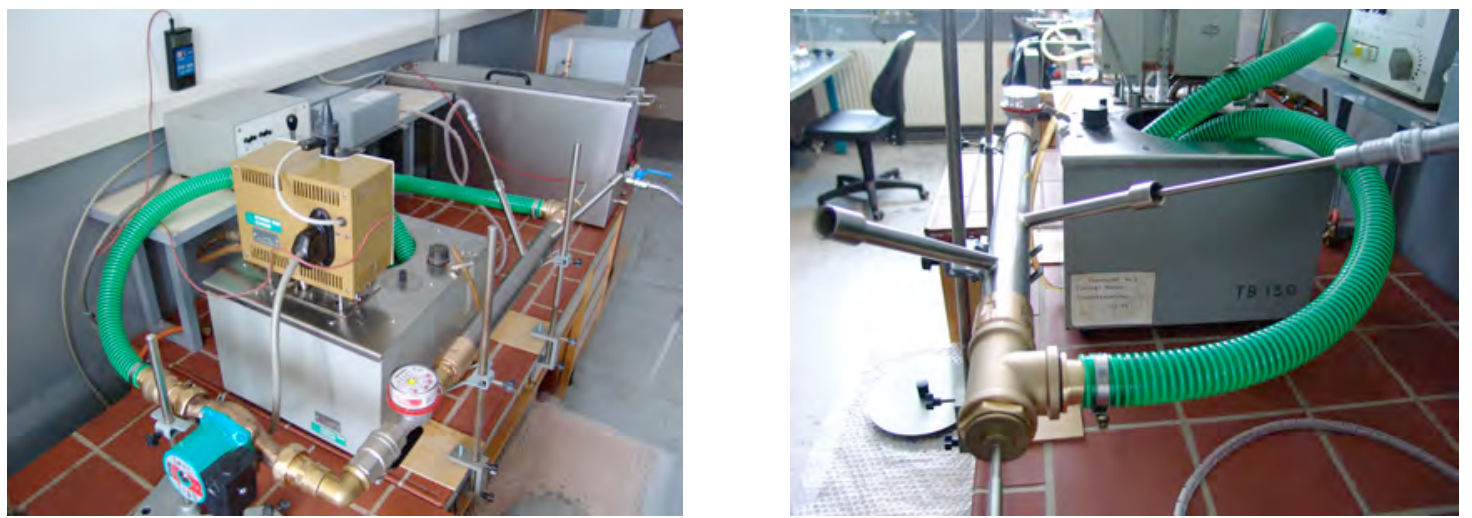

Abbildung 11: Versuchsaufbau mit Wasserkreislauf $\left(T_{M}=50^{\circ} \mathrm{C}, v \approx 0,4 \mathrm{~m} / \mathrm{s}\right)$, im Bild rechts zu sehen: 2 schräg eingebaute Tauchhülsen zur Aufnahme der Messeinsätze der selbstkalibrierenden Thermoelemente 
Als Fixpunktmaterial wurde hier natürlich nicht Zink verwendet, da dieser Fixpunkt zu weit von der Mediumstemperatur entfernt ist. Verwendet wurde eine Wismut-Indium-Legierung, deren Schmelzpunkt bei ca. $73^{\circ} \mathrm{C}$ liegt. Die Messeinsätze wurden in den Wasserkreislauf eingebaut und mehrere Heizzyklen gefahren (maximale Heizleistung $P=11 \mathrm{~W}$, Anstiegsgeschwindigkeit $1 \mathrm{~W} / \mathrm{min}$, dieses Messregime diente als Grundlage der numerischen Berechnungen).

Die folgenden Diagramme zeigen einen Ausschnitt der dabei gemessenen Temperaturverläufe.

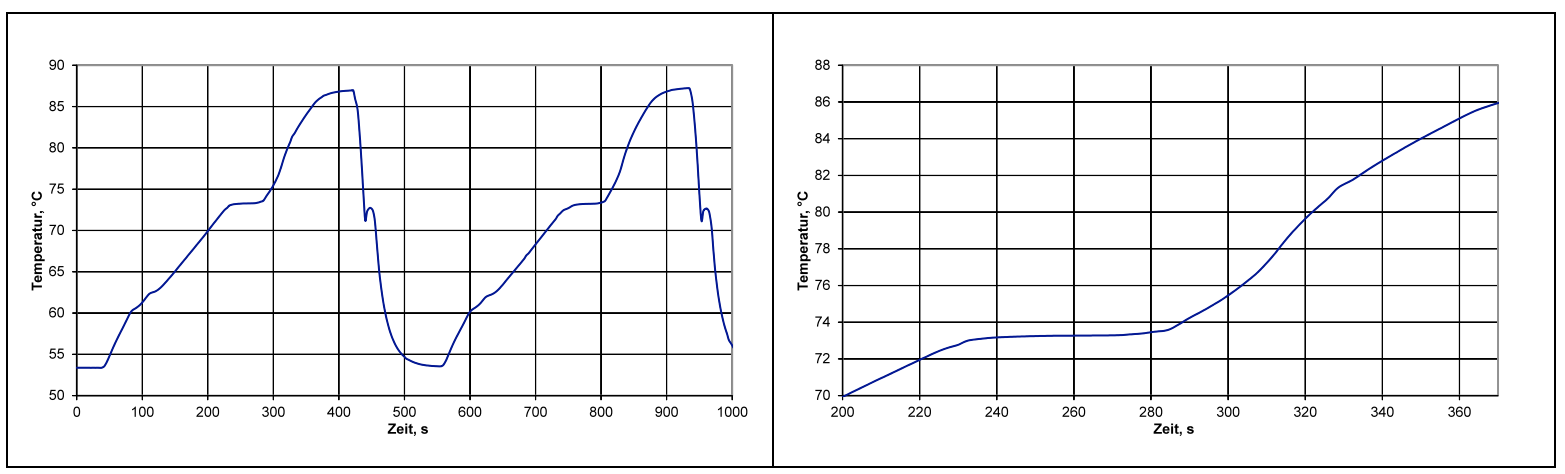

Abbildung 12: Temperaturverlauf im Messeinsatz mit integrierter Miniaturfixpunktzelle (Bi67-In33-Legierung)

In den numerischen Berechnungen konnte dieser Anwendungsfall nicht exakt modelliert werden, da für die verwendete Legierungen die für die Berechnung notwendigen Materialdaten nicht ermittelt werden konnten. Deshalb wurden als Kompromiss Berechnungen auf der Grundlage des oben beschriebenen FEM-Modells mit Indium als Fixpunktmaterial durchgeführt. Der im folgenden Diagramm dargestellte Temperaturverlauf zeigt, dass Plateauverlauf und -dauer mit den experimentellen Ergebnissen annähernd übereinstimmen.

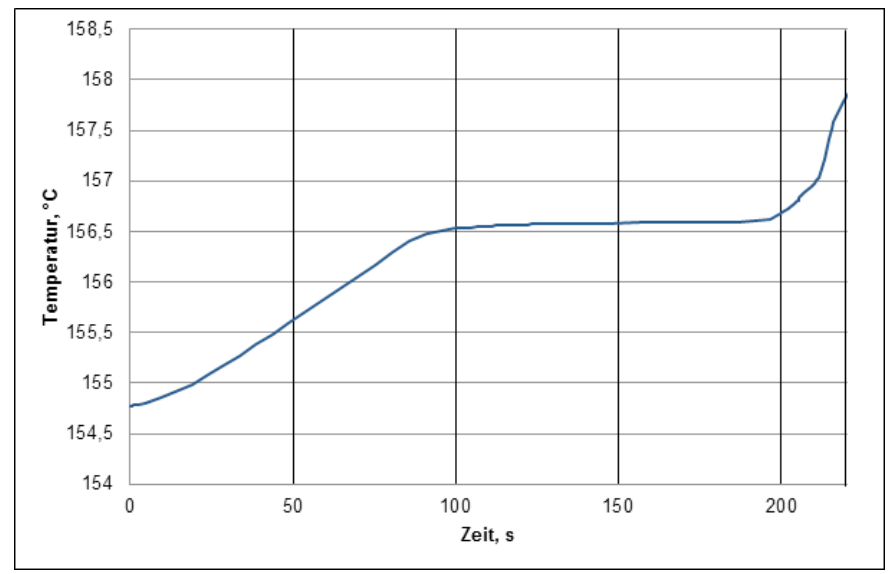

Abbildung 13: Berechneter Temperaturverlauf für Fixpunktmaterial Indium

Diese ersten Ergebnisse zeigen, dass das oben dargestellte numerische Modell prinzipiell den vom Auftraggeber electrotherm gegebenen Anforderungen entspricht.

\section{Zusammenfassung}

Im vorliegenden Beitrag wurde ein Forschungsprojekt zwischen der TU IImenau, Institut für Prozessmess- und Sensortechnik und der Firma electrotherm Geraberg GmbH beschrieben. Die electrotherm $\mathrm{GmbH}$ ist ein mittelständisches Thüringer Unternehmen, das sich auf die Entwicklung und Fertigung von Widerstandsthermometern und Thermoelementen für ein breites Anwenderspektrum aus Industrie, Forschung und Entwicklung spezialisiert hat. Neben den Standardausführungen gehören Temperaturfühler mit speziellen Zulassungen und kundenspezifische Sonderanfertigungen ebenso zum Lieferprogramm wie auch Präzisionsthermometer. 
Der in den bisherigen Kapiteln beschriebene Lösungsweg bildet eine gute Grundlage für die noch durchzuführenden numerischen Berechnungen und experimentellen Verifikationen. Es ist geplant, weitere Variationsrechnungen mit verschiedenen Eintauchtiefen, Materialien und Randbedingungen (Isolation, Geschwindigkeit/Material des Mediums) durchzuführen. Außerdem muss das verwendete Heizregime optimiert werden.

Die experimentelle Untersuchungen sollen unter den geplanten Einsatzbedingungen, vor allem aber auch bei höheren Temperaturen durchgeführt werden, um die Abhängigkeit des statischthermischen Messfehlers und der Form und Länge der Phasenumwandlungsplateaus von der Temperatur, dem Heizregime und den Umgebungsbedingungen zu bestimmen.

Durch die bisherigen Untersuchungen konnte die prinzipielle Eignung der bisherigen Bauformen der selbstkalibrierenden Thermoelemente auch für den Einsatz außerhalb des Kraftwerksbereich nachgewiesen werden.

\section{Literatur}

[1] Bernhard, F.; Augustin, S.; Mammen, H.: „Application of self-calibrating thermometers with miniature fixed-point cells in a temperature range from 300 degree celsius to 650 degree celsius", TEMPMEKO 2004, Dubrovnik, 2004.

[2] Augustin, S., Bernhard, F., Boguhn, D., et al., "Miniature Fixed-Point Thermocouples Applicable for Industrial Purposes", 8th International Symposium on Temperature and Thermal Measurements in Industry and Science TEMPMEKO Berlin, Vol. 1, edited by B. Fellmuth et al., VDE Verlag Berlin, 2001

[3] Krapf, G.; Bernhard, F.; Augustin, S.; Mammen, H.: „Binäre eutektische Aluminium-Legierungen als Materialien in miniaturisierten Fixpunktzellen“; Temperatur 2006, PTB Berlin, Tagungsband

[4] Fixpunkt-Thermoelement-Messgerätesystem SKTE2, Produktinformation 04/2008, Fa. electrotherm $\mathrm{GmbH}$, Geraberg (www.electrotherm.de)

[5] Fixed-point Thermocouples in Power Plants - Long-Term Operational Experiences, International Journal of Thermophysics (2010) 31:1599-1607, DOI 10.1007/s10765-010-0796-7

[6] Hohmann, M.: „Fixpunktthermometer für kleine Rohrquerschnitte“; Abschlussbericht Projektseminar, TU IImenau, Institut für Prozessmess- und Sensortechnik, September 2011

[7] Boguhn,D.: „Miniatur-Fixpunktzellen als Basis selbstkalibrierender elektrischer Berührungsthermometer", Dissertation, Fakultät für Informatik und Automatisierung, TU IImenau, 2002

[8] Barin, Ihsan; Sauert, Fried: „Thermochemical data of pure substances“. Weinheim: VCH-Verlag, 1989

[9] Verein Deutscher Ingenieure; VDI-Gesellschaft Verfahrenstechnik und Chemieingenieurwesen (Hrsg.): „VDI Wärmeatlas: Berechnungsblätter für den Wärmeübergang“. Berlin: Springer 2002

[10] Bernhard, F.; Augustin, S.; Mammen, H.; Donine, A.; Boguhn, D.; Serres, B.; Höfle, F.-A.: „Einsatzerfahrungen mit Selbstkalibrierenden Thermoelementen in Kraftwerken“; Temperatur 2003, PTB Berlin, Tagungsband 